\title{
Spontaneous emission of phonons by coupled quantum dots
}

\author{
T. Brandes, B. Kramer \\ University of Hamburg, 1. Inst. Theor. Physik, Jungiusstr. 9, D-20355 Hamburg, Germany
}

(April 3, 2018)

\begin{abstract}
We find an interference effect for electron-phonon interactions in coupled semiconductor quantum dots that can dominate the nonlinear transport properties even for temperatures close to zero. The intradot electron tunnel process leads to a 'shake up' of the phonon system and is dominated by a doubleslit-like interference effect of spontaneously emitted phonons. The effect is closely related to subradiance of photons in a laser-trapped two-ion system and explains the oscillations in the nonlinear current-voltage characteristics of coupled dots observed recently.
\end{abstract}

PACS: 73.23.Hk, 71.38.+i, $42.50 \mathrm{Fx}$

Spontaneous emission is one of the fundamental concepts of quantum mechanics that can be traced back to such early works as that of Albert Einstein 11. An excited state of a single atom decays exponentially due to the coupling to photons. In a system of two atoms interacting via the common photon field, the decay splits into a sub- and a superradiant channel. This effect is a precursor of the famous Dicke superradiance phenomenon [2] and was verified experimentally in the spontaneous emission of photons from two trapped ions only three years ago [3].

Recently, in a completely different physical system, the emission of phonons from two artificial atoms has been observed 肺. Here, the coupling to the phonon degrees of freedom turned out to dominate the non-linear electron transport through semiconductor double quantum dots even at $m K$ temperatures.

Double quantum dots are well-defined artificial systems for the study of interaction $[5$ and coherent timedependent [6] effects. Here, we propose a theory for a new non-linear transport effect in double quantum dots which corresponds to the Dicke effect, i.e. the collective decay of real atoms. In our theory, the tunneling of single electrons through coupled artificial atoms is renormalized by the interaction with piezoelectric acoustic phonons and leads to an orthogonality catastrophe of the phonon bath if an electron tunnels between the dots. This 'boson shake up' effect [7,8] is determined by an effective density of states $\rho(\omega)$ of the phonon modes $\mathbf{Q}$ that couple to the tunnel process. These interfere like in a double slit experiment when interacting with the electron densities in the two dots. As a result, $\rho(\omega)$ shows oscillations on a scale $\omega_{d}:=c_{s} / d$, where $c_{s}$ is the speed of sound and $d$ the distance between the centers of the two dots. It turns out that the non-linear current peak as a function of the dif- ference $\varepsilon$ between the two relevant many-particle energies is determined by the shape of $\rho(\omega=\varepsilon / \hbar)$. Furthermore, this quantity is analogous to the rate for emission of subradiant photons from two laser-trapped ions [3], when $c_{s}$ is replaced by the speed of light and $d$ by the distance of the ions. Thus, both phenomena are physically closely related. This provides the microscopic mechanism for the oscillations observed recently in a double dot current spectrum 顿. We predict that future experiments with artificial atoms can exploit this analogy to real atoms in more detail. In particular, coherent effects such as superradiance [9] can be manipulated by gate-voltages and external leads in such systems 10 .

In our model, we consider a double quantum dot consisting of a left and a right dot ( $\mathrm{L}$ and $\mathrm{R}$, respectively) which are connected via a tunnel barrier. Each dot is connected to an electron reservoir in thermal equilibrium with chemical potentials $\mu_{L}$ (source) and $\mu_{R}<\mu_{L}$ (drain). For the physical phenomena we are interested in, it is sufficient [11] to restrict the dot Hilbert space to the three states $|0\rangle=\left|N_{L}, N_{R}\right\rangle,|L\rangle=\left|N_{L}+1, N_{R}\right\rangle$, and $|R\rangle=\left|N_{L}, N_{R}+1\right\rangle$, which correspond to manyparticle ground states with $N_{L}(+1)$ electrons in the left and $N_{R}(+1)$ electrons in the right dot. The corresponding ground state energies $\varepsilon_{L}$ of $|L\rangle$ and $\varepsilon_{R}$ of $|R\rangle$ are in the window $\mu_{L}>\varepsilon_{L}, \varepsilon_{R}>\mu_{R}$. We assume that the Coulomb charging energy $U_{c}$ is the largest energy scale, and it is not possible to charge the double dot with more than one additional electron. In [4], no enhanced tunnel current was observed for $\varepsilon:=\varepsilon_{L}-\varepsilon_{R}<0$ at low temperatures so that excited many-body states play no role. In particular, $U_{c} \sim 1 \mathrm{meV}$ was one order of magnitude larger than the external source drain voltage $V_{S D}$. This situation has to be contrasted with the regime $V_{S D} \gtrsim U_{c}$ (absence of blockade effects, [12]).

We define operators $n_{L}=|L\rangle\left\langle L\left|, n_{R}=\right| R\right\rangle\langle R|, p=$ $|L\rangle\left\langle R\left|, s_{L}=\right| 0\right\rangle\left\langle L\left|, s_{R}=\right| 0\right\rangle\langle R|$, and the total system Hamiltonian $H$ as the sum of the dot, the phonon, the reservoir and the electron-phonon interaction

$$
\begin{aligned}
H & =H_{0}^{\prime}+H_{T}+H_{V}+H_{\alpha \beta} \\
H_{0}^{\prime} & =\varepsilon_{L} n_{L}+\varepsilon_{R} n_{R}+H_{p}+H_{r e s} \\
H_{T} & =T_{c}\left(p+p^{\dagger}\right), \quad H_{p}=\sum_{\mathbf{Q}} \omega_{\mathbf{Q}} a_{\mathbf{Q}}^{\dagger} a_{\mathbf{Q}} \\
H_{\alpha \beta} & =\sum_{\mathbf{Q}}\left(\alpha_{\mathbf{Q}} n_{L}+\beta_{\mathbf{Q}} n_{R}\right)\left(a_{-\mathbf{Q}}+a_{\mathbf{Q}}^{\dagger}\right) \\
H_{V} & =\sum_{\mathbf{k}}\left(V_{\mathbf{k}} c_{\mathbf{k}}^{\dagger} s_{L}+W_{\mathbf{k}} d_{\mathbf{k}}^{\dagger} s_{R}+c . c .\right)
\end{aligned}
$$




$$
H_{r e s}=\sum_{\mathbf{k}} \varepsilon_{\mathbf{k}}^{L} c_{\mathbf{k}}^{\dagger} c_{\mathbf{k}}+\sum_{\mathbf{k}} \varepsilon_{\mathbf{k}}^{R} d_{\mathbf{k}}^{\dagger} d_{\mathbf{k}} .
$$

Here, the tunneling between left and right dots is described by a single tunnel matrix element $T_{c}$. In the standard tunnel Hamiltonian $H_{V}, V_{\mathbf{k}}$ and $W_{\mathbf{k}}$ couple the dot to a continuum of channels $\mathbf{k}$ of the left and right electron reservoir $H_{\text {res }}$. The spin of the electron does not play any role here and is suppressed. The term $H_{p}$ describes the lattice vibrations in harmonic approximation; the creation operator for a phonon of mode $\mathbf{Q}$ is $a_{\mathbf{Q}}^{\dagger}$. The electron-phonon matrix elements are defined by $\alpha_{\mathbf{Q}}:=\lambda_{\mathbf{Q}}\left\langle L\left|e^{i \mathbf{Q r}}\right| L\right\rangle$ and $\beta_{\mathbf{Q}}:=\lambda_{\mathbf{Q}}\left\langle R\left|e^{i \mathbf{Q} \mathbf{r}}\right| R\right\rangle$, where $\lambda_{\mathbf{Q}}$ is the matrix element for the interaction of 2DEG electrons and phonons. We have already neglected the nondiagonal term of the electron-phonon interaction which contains matrix elements $\gamma_{\mathbf{Q}}:=\lambda_{\mathbf{Q}}\left\langle L\left|e^{i \mathbf{Q r}}\right| R\right\rangle$. We have checked in a seperate master equation calculation that such terms modify only weakly the tunnel current and do not lead to the oscillatory phenomena observed in [4, which is due to the non-perturbative shake-up process that we describe in the following.

Suppose an electron tunnels between two regions of space $(L$ and $R)$ and interacts with a phonon field. With the interaction of the form $H_{\alpha \beta}$, Eq.(1), the electronphonon coupling locally changes the energy of the electron, depending on whether it is in $L$ or in $R$. During tunneling, its wave function experiences an additional a phase shift $e^{i \phi}$ due to this coupling. This phase shift is zero if the coupling is identical in both regions, i.e. if $\alpha_{\mathbf{Q}}=\beta_{\mathbf{Q}}$. If, as we show below, $\alpha_{\mathbf{Q}} \neq \beta_{\mathbf{Q}}$, the phase shift depends on the state of the phonon field. From the point of view of the phonons, initial (before the tunneling) and final (after tunneling) phonon states are no longer the same. From the point of view of the electron, the effective tunnel amplitude changes non-trivially. In particular, it becomes time-dependent.

We introduce now a unitary polaron transformation 113] of the Hamiltonian that naturally leads to the phase factors mentioned above. For any operator $O$, we define $\bar{O}:=e^{S} O e^{-S}, S:=n_{L} A+n_{R} B$ with $A:=$ $\sum_{\mathbf{Q}}\left(1 / \omega_{\mathbf{Q}}\right) \quad\left(\alpha_{\mathbf{Q}} a_{\mathbf{Q}}^{\dagger}-\alpha_{-\mathbf{Q}} a_{\mathbf{Q}}\right)$ and $B:=\sum_{\mathbf{Q}}\left(1 / \omega_{\mathbf{Q}}\right)$ $\left(\beta_{\mathbf{Q}} a_{\mathbf{Q}}^{\dagger}-\beta_{-\mathbf{Q}} a_{\mathbf{Q}}\right)$. This leads to renormalized energies $\overline{\varepsilon_{L}}=\varepsilon_{L}-\sum_{\mathbf{Q}}\left|\alpha_{\mathbf{Q}}\right|^{2} / \omega_{\mathbf{Q}}$ and $\overline{\varepsilon_{R}}=\varepsilon_{R}-\sum_{\mathbf{Q}}\left|\beta_{\mathbf{Q}}\right|^{2} / \omega_{\mathbf{Q}}$, and a renormalized intra-dot tunneling Hamiltonian $\bar{H}_{T}=T_{c}\left(p X+p^{\dagger} X^{\dagger}\right)$. Here, the phase operator $X=$ $\prod_{\mathbf{Q}} D_{\mathbf{Q}}\left(\left(\alpha_{\mathbf{Q}}-\beta_{\mathbf{Q}}\right) / \omega_{\mathbf{Q}}\right)$ is the product of unitary displacement operators $D_{\mathbf{Q}}(z):=\exp \left(z a_{\mathbf{Q}}^{\dagger}-z^{*} a_{\mathbf{Q}}\right)$, where the operation of $D_{\mathbf{Q}}(z)$ on the boson vacuum creates a coherent state of the boson field mode $\mathbf{Q}$. The factors $X$ and $X^{\dagger}$ in the tunnel Hamiltonian $\bar{H}_{T}$ drastically change the transport properties of the double dot.

We assume that the coupling to the left and right electron reservoirs is weak such that a standard Born and Markov approximation holds. In contrast to this, we calculate to all orders of the intradot tunneling $T_{c}$ and the electron-phonon coupling because the renormalization of the tunneling by the phase factors $X$ is a nonperturbative effect. From the Liouville equation for the total density matrix of the system, one obtains the equations of motion (cp. 11, 14])

$$
\begin{aligned}
& \frac{d}{d t}\left\langle n_{L}\right\rangle_{t}=-i T_{c}\left(\langle p\rangle_{t}-\left\langle p^{\dagger}\right\rangle_{t}\right)+2 \Gamma_{L}\left(1-\left\langle n_{L}\right\rangle_{t}-\left\langle n_{R}\right\rangle_{t}\right) \\
& \frac{d}{d t}\left\langle n_{R}\right\rangle_{t}=i T_{c}\left(\langle p\rangle_{t}-\left\langle p^{\dagger}\right\rangle_{t}\right)-2 \Gamma_{R}\left\langle n_{R}\right\rangle_{t} \\
&\langle p\rangle_{t}=-\Gamma_{R} \int_{0}^{t} d t^{\prime} e^{i \varepsilon\left(t-t^{\prime}\right)}\left\langle X_{t} X_{t^{\prime}}^{\dagger} \tilde{p}\left(t^{\prime}\right)\right\rangle_{t^{\prime}} \\
&-i T_{c} \int_{0}^{t} d t^{\prime} e^{i \varepsilon\left(t-t^{\prime}\right)}\left(\left\langle n_{L} X_{t} X_{t^{\prime}}^{\dagger}\right\rangle_{t^{\prime}}-\left\langle n_{R} X_{t^{\prime}}^{\dagger} X_{t}\right\rangle_{t^{\prime}}\right),
\end{aligned}
$$

where $\Gamma_{L}:=2 \pi \sum_{\mathbf{k}} V_{\mathbf{k}}^{2} \delta\left(\bar{\varepsilon}_{L}-\varepsilon_{\mathbf{k}}^{L}\right), \Gamma_{R}:=2 \pi \sum_{\mathbf{k}} W_{\mathbf{k}}^{2}$ $\delta\left(\bar{\varepsilon}_{R}-\varepsilon_{\mathbf{k}}^{R}\right)$, and the chemical potential $\mu_{L}\left(\mu_{R}\right)$ of the left (right) electron reservoir is assumed to be far above (below) $\bar{\varepsilon}_{L}\left(\bar{\varepsilon}_{R}\right)$ so that no electrons can tunnel from the left dot into the left reservoir, or from the right reservoir into the right dot. Furthermore, $\varepsilon=\bar{\varepsilon}_{L}-\bar{\varepsilon}_{R}$, where we neglect the difference in the energy renormalization in both dots, $\tilde{p}(t)=p e^{i \varepsilon t} X_{t}$, and $X_{t}$ denotes the timeevolution of $X$ with $H_{p}$.

We assume the phonon system to be in thermal equilibrium at all times. We use a decoupling of the reduced density matrix $\tilde{\rho}\left(t^{\prime}\right)$ of the dot, $\tilde{\rho}\left(t^{\prime}\right) \approx \rho_{p h}^{0} \operatorname{Tr}_{p h}\left\{\tilde{\rho}\left(t^{\prime}\right)\right\}$. We introduce the Laplace transform

$$
C_{\varepsilon}(z):=\int_{0}^{\infty} d t e^{-(z-i \varepsilon) t}\left\langle X_{t} X_{0}^{\dagger}\right\rangle_{0}
$$

and $n_{L}(z):=\int_{0}^{\infty} d t e^{-z t}\left\langle n_{L}\right\rangle_{t}$ etc. for $z>0$. By transforming Eq.(2) into $z$-space, the expectation value of the current operator $\hat{I}:=i T_{c}\left(p-p^{\dagger}\right)$ (we set the electron charge $e=1$ for convenience), can be obtained in the stationary limit $t \rightarrow \infty$ from the $1 / z$-coefficient of the $I(z)$-expansion into a Laurent series for $z \rightarrow 0$. The result is

$$
\begin{gathered}
\langle I\rangle_{t \rightarrow \infty}=T_{c}^{2} \frac{2 \Re\left(C_{\varepsilon}\right)+2 \Gamma_{R}\left|C_{\varepsilon}\right|^{2}}{\left|1+\Gamma_{R} C_{\varepsilon}\right|^{2}+2 T_{c}^{2} B_{\varepsilon}} \\
B_{\varepsilon}:=\Re\left\{\left(1+\Gamma_{R} C_{\varepsilon}\right)\left[\frac{C_{-\varepsilon}}{2 \Gamma_{R}}+\frac{C_{\varepsilon}^{*}}{2 \Gamma_{L}}\left(1+\frac{\Gamma_{L}}{\Gamma_{R}}\right)\right]\right\} .
\end{gathered}
$$

Here, $C_{\varepsilon}=C_{\varepsilon}(\delta \rightarrow 0)$. One can verify that in the limit of no phonon coupling, $C_{\varepsilon}=i / \varepsilon$, thus obtaining a simple Lorentzian curve for the stationary tunnel current as a function of $\varepsilon$ [1]. The appearance of $T_{c}$ in the denominator of Eq.(4) indicates that this result is nonperturbative, i.e. valid to all orders in $T_{c}$. The modification of this curve by the electron-phonon interaction is completely described by the form of $C_{\varepsilon}$ which we will discuss now.

For a harmonic phonon system in thermal equilibrium at temperature $T$, one finds the correlation function $\left\langle X_{t} X_{0}^{\dagger}\right\rangle_{0}=e^{-\Phi\left(t-t^{\prime}\right)}$ with 
$\Phi(t):=\int_{0}^{\infty} d \omega \rho(\omega)\left\{(1-\cos \omega t) \operatorname{coth} \frac{\hbar \omega}{2 k_{B} T}+i \sin \omega t\right\}$

$\rho(\omega)=\sum_{\mathbf{Q}} \frac{\left|\alpha_{\mathbf{Q}}-\beta_{\mathbf{Q}}\right|^{2}}{\omega^{2}} \delta\left(\omega-\omega_{\mathbf{Q}}\right)$.

The matrix elements $\alpha_{\mathbf{Q}}$ and $\beta_{\mathbf{Q}}$ are $\alpha_{\mathbf{Q}}=$ $\lambda_{\mathbf{Q}} \int d^{3} \mathbf{x} e^{i \mathbf{Q} \mathbf{x}} \rho_{L}(\mathbf{x}), \beta_{\mathbf{Q}}=\lambda_{\mathbf{Q}} \int d^{3} \mathbf{x} e^{i \mathbf{Q} \mathbf{x}} \rho_{R}(\mathbf{x})$, with the local electron densities $\rho_{L}(\mathbf{x})$ and $\rho_{R}(\mathbf{x})$ in the left and the right dot, respectively. The exact form of both $\rho_{L}(\mathbf{x})$ and $\rho_{R}(\mathbf{x})$ depends on the shape of the dots and on the number of electrons $N_{L}$ and $N_{R}$. In the stationary state for $t \rightarrow \infty$, both densities can be assumed to be smooth functions of $\mathbf{x}, \rho_{L}(\mathbf{x}) \approx \rho_{e}\left(\mathbf{x}-\mathbf{x}_{L}\right), \quad \rho_{R}(\mathbf{x}) \approx$ $\rho_{e}\left(\mathbf{x}-\mathbf{x}_{R}\right)$, where we have assumed that both left and right electron densities are described by the same profile. Here, $\rho_{e}(\mathbf{x})$ is relatively sharply peaked around zero. One obtains $\alpha_{\mathbf{Q}}=\lambda_{\mathbf{Q}} e^{i \mathbf{Q r}_{L}} P(\mathbf{Q}), \beta_{\mathbf{Q}}=\lambda_{\mathbf{Q}} e^{i \mathbf{Q} \mathbf{r}_{R}} P(\mathbf{Q})$, where $P(\mathbf{Q})=\int d^{3} \mathbf{x} e^{i \mathbf{Q} \mathbf{x}} \rho_{e}(\mathbf{x})$. Therefore, $\beta_{\mathbf{Q}}=\alpha_{\mathbf{Q}} e^{i \mathbf{Q d}}$ with $\mathbf{d}=\mathbf{r}_{R}-\mathbf{r}_{L}$. The matrix elements $\alpha_{\mathbf{Q}}$ and $\beta_{\mathbf{Q}}$ thus coincide up to the phase factor $e^{i \mathbf{Q d}}$. This phase factor is the origin of the oscillations in the effective density of states $\rho(\omega)$ of phonon modes that couple to the tunnel process. We note that the explicit form of $\alpha_{\mathbf{Q}}$ and $\beta_{\mathbf{Q}}$ can be calculated exactly when the two states $|L\rangle$ and $|R\rangle$ are few particle wave functions.

For sharp charge density profiles with Fourier transform $P(\mathbf{Q}) \rightarrow 1$, we find

$$
\rho(\omega) \approx \frac{g}{\omega}\left(1-\frac{\omega_{d}}{\omega} \sin \left(\frac{\omega}{\omega_{d}}\right)\right) e^{-\omega / \omega_{c}}, \quad g=\frac{\lambda^{2}}{\pi^{2} \hbar^{2} c_{s}^{3}},
$$

where $\omega_{d}:=c_{s} / d, d=|\mathbf{d}|, c_{s}$ is the longitudinal speed of sound, and a smooth exponential cutoff $\omega_{c}$ is an effective Debye frequency. The latter is due to the finite extension $l$ of the wave functions in the dots leading to a cutoff of phonons with wave vectors $Q=|\mathbf{Q}| \gtrsim 1 / l$. The phonons are assumed to be piezoelectric acoustical bulk (three-dimensional) modes with an interaction matrix element $\left|\lambda_{Q}\right|^{2}=\lambda^{2} / V c_{s} Q, \lambda^{2}=\hbar P / 2 \rho_{M}$, where $\omega_{Q}=c_{s} Q$ is the phonon dispersion, $V$ the volume and $\rho_{M}$ the mass density of the crystal, and $P$ the piezoelectric coupling constant. Dynamical screening effects of the 2DEG have been absorbed into the value of $P$. Using typical GaAs parameters [15], we obtain $g \approx 0.05$.

The most important feature of $\rho(\omega)$, Eq.(6), are the oscillations on the scale $\omega_{d}=c_{s} / d$, (Fig. 近, inset). In fact, these lead to the oscillations in the current profile due to phonon emission in the experiment [1]: Fig. 1] shows the stationary current Eq.(4) at different temperatures for parameters close to the ones of the experiment. With $d=200 \cdot 10^{-9} \mathrm{~m}$ and $c_{s}=5000 \mathrm{~m} / \mathrm{s}$, we obtain $\hbar \omega_{d}=16.5 \mu \mathrm{eV}$, which is the scale on which the oscillations occur in [1. The cutoff energies are assumed as $\hbar \omega_{c}=1 \mathrm{meV}$ and $\hbar \delta=1 \mu \mathrm{eV}$. A small but finite value of $\delta$ in $C_{\varepsilon}(\delta \rightarrow 0)$ simulates off-diagonal electron-phonon processes which are not included in $\rho(\omega)$ for $\hbar \omega=\varepsilon \rightarrow 0$. At low temperatures, there is a broad oscillatory shoulder for $\varepsilon>0$ (spontaneous phonon emission). It reflects the oscillations in $\rho(\omega)$ which in turn determines $C_{\varepsilon}$ in Eq. (4). Its real part $\Re C_{\varepsilon}$ is proportional to the probability density for inelastic tunneling from the left to the right dot with energy transfer $\varepsilon$ 8]. In the limit $\omega_{d}=0$, one finds $\Re C_{\varepsilon}=(2 \pi / \Gamma(g)) \varepsilon^{g-1} e^{-\varepsilon} \theta(\epsilon)$ at $T=0$, where only spontaneous phonon emission is possible. This shows that the effect is non-perturbative in the electron-phonon coupling $g$.

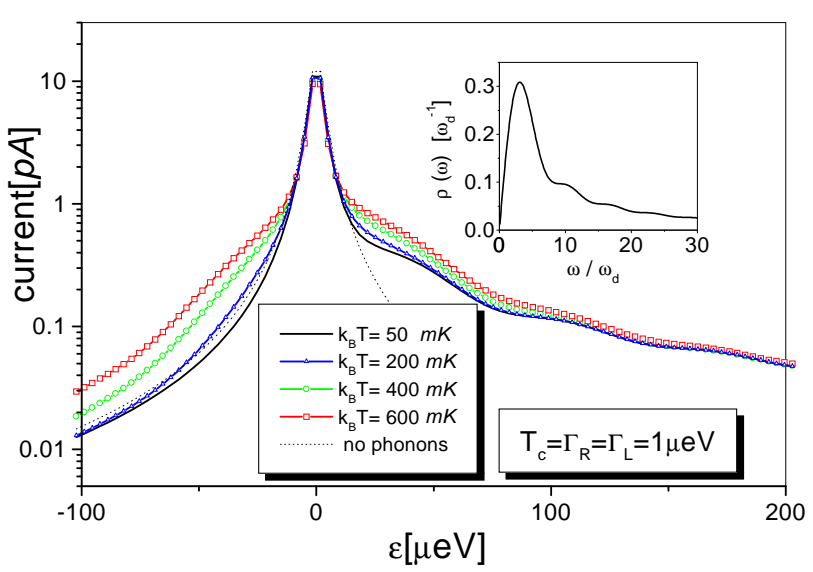

FIG. 1. Stationary tunnel current, Eq.(4), as a function of the energy difference $\varepsilon$ between left and right dot ground state energies. Dimensionless electron-phonon coupling parameter: $g=0.05$. Inset: effective density of states $\rho(\omega)$ of phonon modes, Eq.(5) and Eq.(6).

As an important consequence, even for small coupling constants $g$ and $T \rightarrow 0$, the electron-phonon interaction can dominate the non-linear electron transport. Furthermore, we find an energy dependence of the current for $\varepsilon \gtrsim 50 \mu \mathrm{eV}$ between $1 / \varepsilon$ (larger $g$ ) and $1 / \varepsilon^{2}$ (smaller $g$ ), depending on $g$. Note that the tunnel current $I$ is determined by the complex function $C_{\varepsilon}$ which contains all effects of energy renormalization due to the electronphonon coupling. At higher temperatures, the current at the absorption side $\varepsilon<0$ increases faster than at the emission side where the oscillations start to be smeared out. For $\varepsilon<0$ a shoulder-like structure appears, consistent with the observation in [4].

We note that our results are consistent with a previous model [16] for inelastic tunneling via pairs of impurities. There, perturbation theory in the electron-phonon deformation potential coupling was used to obtain the nonlinear current (without oscillations) for large area tunnel junctions after averaging over an ensemble of impurity pairs. 
The spontaneous emission of phonons in double dots is closely analogous to the subradiant spontaneous decay by emissions of photons from a laser-trapped two-ion system [3]. There, the interaction of the atomic dipoles $\hat{\mathbf{d}}_{i}$, at positions $\mathbf{r}_{i}, i=1,2$ with the electromagnetic field has the form 17

$$
H_{e p h}=\sum_{\mathbf{Q} s} \mathbf{g}_{\mathbf{Q} s}\left(\hat{\mathbf{d}}_{1} \exp i \mathbf{Q} \mathbf{r}_{1}+\hat{\mathbf{d}}_{2} \exp i \mathbf{Q} \mathbf{r}_{2}\right)
$$

with $\mathbf{g}_{\mathbf{Q} s}=-i(2 \pi c Q / V)^{1 / 2} \vec{\varepsilon}_{\mathbf{Q}, s}$ with the speed of light $c$, and polarization vectors $\vec{\varepsilon}_{\mathbf{Q}, s}$ for polarization direction $s$. This leads to rates for super- and subradiant decay of the form [9] $\Gamma(Q)_{ \pm}=\Gamma_{0}(Q)[1 \pm \alpha \sin (Q d) /(Q d)]$ with $\alpha=1$ $(\alpha=3 / 2)$ if the vector character of the light is (not) neglected, $Q=\omega / c$, and $\Gamma_{0}(Q) \propto Q^{3}$. The subradiant channel $(-)$ is due to the decay of the singlet state that corresponds to the difference $\hat{\mathbf{d}}_{1} \exp i \mathbf{Q} \mathbf{r}_{1}-\hat{\mathbf{d}}_{2} \exp i \mathbf{Q} \mathbf{r}_{2}$ of the dipole moments. The interaction with a phonon of mode $\mathbf{Q}$ in the double dot is $\propto n_{L} \exp i \mathbf{Q} \mathbf{r}_{1}+n_{R} \exp i \mathbf{Q} \mathbf{r}_{2}$. Thus, it has the same 'interference form' as in the twoion case, Eq.(7). The tunnel current is modified by the phase difference of the electron before and after tunneling whence $\rho(\omega)$, Eq.(16), corresponds to the subradiant rate $\Gamma_{-}$. Although the microscopic mechanism is different in both cases (for light the rates $\Gamma_{0}$ are $\propto \omega^{3}$, for piezoelectric phonons $\propto 1 / \omega)$, the interference term $\sin (Q d) / Q d$ in both cases is due to the 'interference of matrix elements' in the interaction Hamiltonian and has the same physical origin.

In the atom-trap experiment, the Dicke effect, i.e. the existence of two different radiation channels $\Gamma_{ \pm}$, has been verified by changing the ion distance. The experimental data of the double quantum dot [4] seem to indicate that for larger distance $d$ of the dots the oscillations $\propto \sin \left(\varepsilon / \hbar \omega_{d}\right)$ as a function of $\varepsilon$ become faster which is consistent with $\omega_{d}=c_{s} / d$ in Eq.(6).

In conclusion, we have found an interference effect which explains the recently observed phonon emission spectrum in the transport through coupled quantum dots. The analogy between artificial and real multiple atom systems allows to speculate about further realizations of coherent optical effects in semiconductor quantum dot experiments, similar to the oscillatory Dicke effect we have found recently [10]. In the case of phonons discussed here, the coherence (oscillations due to interference) showed up within a dissipative process, i.e. spontaneous emission. In order to further study such 'coherent dissipative phenomena', we suggest systems of two of more double quantum dots which are coupled via the common phonon field. The non-linear transport properties are then determined by the electron-phonon coupling both within each and between the double dots.

This work has been supported by the TMR network 'Quantum transport in the frequency and time domains', the Graduiertenkolleg 'nanostrukturierte Festkörper'
(Hamburg), the SFB 508 'Quantenmaterialien' (Hamburg), and the DFG project Kr 627/9-1.

[1] A. Einstein, Phys. Z. 18, 121 (1917).

[2] R. H. Dicke, Phys. Rev. 93, 99 (1954).

[3] R. G. DeVoe and R. G. Brewer, Phys. Rev. Lett. 76, 2049 (1996).

[4] T. Fujisawa et al., Science 282, 932 (1998).

[5] K. A. Matveev, L. I. Glazman, and H. U. Baranger, Surf. Scie. 361, 623 (1996); C. A. Stafford, R. Kotlyar, and S. Das Sarma, Phys. Rev. B 58, 7091 (1998) .

[6] T. Fujisawa and S. Tarucha, Superlatt. Microstr. 21, 247 (1996); R. H. Blick et al., Phys. Rev. Lett. 81, 689 (1998); ibid. 80, 4032 (1998); Ph. Brune, C. Bruder, and H. Schoeller, Phys. Rev. B 56, 4730 (1997) .

[7] S. M. Girvin et al., Phys. Rev. Lett. 64, 3183 (1990).

[8] G.-L. Ingold and Y. V. Nazarov, in Single Charge Tunneling, Vol. 294 of NATO ASI Series B, edited by H. Grabert and M. H. Devoret (Plenum Press, New York, 1991), p. 21.

[9] M. Benedict et al., Super-radiance (Institute of Physics, Bristol, 1996).

[10] T. Brandes, J. Inoue, and A. Shimizu, Phys. Rev. Lett. 80, 3952 (1998).

[11] T. H. Stoof and Yu. V. Nazarov, Phys. Rev. B 53, 1050 (1996).

[12] See M. Raikh, A. Asenov, Superlatt. Microstr. 11, 325 (92), where the crossover to $V_{S D}>U_{c}$ shows up as a step in the $I-V$ curve.

[13] G. D. Mahan, Many-Particle Physics (Plenum Press, New York, 1990).

[14] S. A. Gurvitz and Ya. S. Prager, Phys. Rev. B 53, 15932 (1996).

[15] H. Bruus, K. Flensberg, and H. Smith, Phys. Rev. B 48, 11144 (1993).

[16] L. I. Glazman and K. A. Matveev, Sov. Phys. JETP 67, 1276 (88). Note that there, the expression for the spontaneous emission rate $\gamma$ already contains the differences of phase factors $\left(\sim|1-\exp \{i \mathbf{Q d}\}|^{2}\right.$ in our notation $)$, which are averaged out in the case they consider.

[17] G. S. Argawal, Quantum Statistical Theories of Spontaneous Emission (Springer, Berlin, 1974), Vol. 70. 